Magnetotactic bacteria synthesize nano-sized magnetite particles that are highly consistent in size and morphology within the bacterial species. The particles are individually covered with a thin organic membrane, which confers high and even dispersion in aqueous solutions. Since superior characteristics, in comparison to artificial magnetites, makes them ideal materials for biotechnological applications. Recent molecular studies, including comprehensive proteomic, transcriptomic, and genomic analyses, have considerably improved our hypotheses of the bacterial magnetite formation mechanism. On the basis of these results, it has been described that the mechanism underlying magnetosome formation involves multiple processes including vesicle formation and alignment, vesicular iron accumulation, and iron oxide crystallization. Genome sequence analyses also further revealed that the genes encoding proteins specifically located on the bacterial magnetite surface are conserved within the member of magnetotactic bacteria species in the form of a genomic island. Consequently, some of the genes and proteins identified from these basic studies have allowed us to express functional proteins efficiently onto bacterial magnetite surface, via genetic engineering, permitting the preservation of the expressed protein's activity, leading to a simple preparation of functional protein-magnetic particle complexes. Their application in high-sensitive immunoassay, DNA analysis, drug screening, and cell separation will also be introduced.

\section{SP5-04ＢZ 反応で駆動する化学ロボットの創製}

Development of a novel autonomous chemical robot

Yusuke Hara (1) ((I) Graduate School of Science and Engineering, Waseda Univ.)

Many kinds of stimuli-responsive polymers and gels have been developed and applied to actuators and artificial muscles. In particular, electroactive polymers that change shape when stimulated electrically are promising. In all cases, however, the mechanical motion is driven by external stimuli, for example, reversing the direction of electric field. On the other hand, many living organisms can generate an autonomous motion without external driving stimuli, for example the self-beating heart muscle. Here we show novel bending-stretching, selfwalking and peristaltic motion of polymer gel actuators without switching of external stimuli. The self-oscillating motion is produced by dissipating chemical energy of an oscillating reaction, i.e., the Belousov-Zhabotinsky(BZ) reaction occurring inside the gel. Although the gel is a synthetic polymer, it shows autonomous motion as if it is alive. However, operating conditions were limited to non-physiological environments in the presence of strong acids and oxidants. Therefore, recently, in order to expand the application of those gels to the biomedical field, we attempted the construction of a novel self-oscillating polymer that can cause the self-oscillation under physiological conditions. We synthesized a novel quarternary copolymer which included both $\mathrm{pH}$-control and oxidant-supplying sites in the poly(NIPAAm-co-Ru(bpy)3) chain. As a result, we first succeeded in causing the self-oscillation under biological conditions in the presence of an organic acid (malonic acid) existed. In addition, we demonstrated that an on-off switching of the self-oscillation can be controlled by temperature. Moreover, by utilizing a highly concentrated polymer solution, we succeeded in causing a viscosity self-oscillation for the first time.

\section{SP5-05 昆虫一機械融合システムで探る適応能}

Insect-Machine Hybrid System for Understanding an Adaptive Behavior

Ryohei Kanzaki (1) ((1) Research Center for Advanced Science and Technology, The University of Tokyo)

Insects are the most diverse and abundant animal group representing more than $70 \%$ of all known animal species. They display a diversity of sophisticated behaviors adapted to their environments by the processing of a simple nervous system. Insects will become an excellent model for understanding adaptive control in biological systems. We have developed an insect-machine hybrid system that acts based on the behavioral or the brain neural output of an insect, as a novel experimental system that manipulates the interaction between an insect and the real environment in order to evaluate and understand environmental adaptation. We have used a male silkmoth as a robot controller because the moth exhibits a well-defined pheromone searching behavior, the neural basis of which has been well characterized by our group. The robot measures the behavior of an insect tethered on the robot and moves based on the insect's behavior or the neural activity of the insect brain. Therefore, it is possible to cause changes in the same way as manipulation of the sensory-motor system of the insect by giving arbitrary manipulation to the motion system of the robot. In this lecture, as an example of adaptive behavior of an insect, odor-source orientation behavior and its neural basis will be shown. Then, I will demonstrate the insect-machine hybrid system that will lead to great insight for evaluating and understanding adaptive behaviors, which will inspire control and communication in engineered systems

3SP6-01 固体 NMR による膜タンパク質の構造、ダイナミクス、相互作用 と活性相関の解明

Structure, dynamics and interaction of membrane proteins in relation to the function as revealed by solid-state NMR

\section{Akira Naito (1) ((1) Yokohama National University)}

Membrane proteins play an important role in the cell membrane as receptors, ion channel, transporter in the living systems. To understand the functional insight of the membrane proteins, it is important to analyze not only the structure but also the dynamics of the membrane proteins. First, bacteriorhodopsin (bR) ia a membrane protein from halobacterium salinarum and consists of seven TM $\alpha$ helices and retinal covalently linked to K216 through a protonated Sciff base. Structural changes in bacteriorhodopsin (bR) in two different processes of retinal reconstitution were investigated by observing the ${ }^{13} \mathrm{C}$ and ${ }^{15} \mathrm{~N}$ solid-state NMR spectroscopy. Regenerated $\mathrm{bR}$ was prepared following the addition of retinal into bO. E1001 bR was cultured from a retinal-deficient strain following the addition of retinal to growing cells. ${ }^{15} \mathrm{~N}$ NMR signals at Pro70 in BC-loop in WT-bR and E1001-bR were observed at $122.4 \mathrm{ppm}$, whereas signal was partly suppressed in regenerated $\mathrm{bR}$. This result indicates that the $\mathrm{BC}$ loop may not always fold correctly in the regenerated $b R$. Second, pharaonis phoborhodopsin $(p \mathrm{pR})$ is a negative phototaxis receptor of Natronomonus pharaonis, and forms a complex with cognate transducer $(p \mathrm{HtrII})$. We examined a possible local dynamics change of $p \mathrm{pR}$ and its $\mathrm{D} 75 \mathrm{~N}$ mutant complexed with $p \mathrm{HtrII}$, using solid-state ${ }^{13} \mathrm{C}$ NMR. The local fluctuation frequency at the C-terminal tip was increased when D75N was bound to $p \mathrm{HtrII}$. At the same time, the fluctuation frequency of cytoplasmic portion of $p \mathrm{HtrII}$ is lowered when $p \mathrm{pR}$ is replaced by $\mathrm{D} 75 \mathrm{~N}$ in the complex. This means that the C-terminal tip partly participate in binding with linker region of $p \mathrm{HtrII}$ in the dark, but this portion might be released at the signaling state leading to mutual association of the two transducers in the cytoplasmic regions within the $p \mathrm{pR} / p \mathrm{HtrII}$ complex.

\section{SP6-02 Dynamical NMR Structures and Functional Insights of Cytochrome-b5 and Amyloid Peptides \\ Ayyalusamy Ramamoorthy (1) ((1) University of Michigan)}

Most of the drugs in use today are metabolized in part by a small set of human cyt P450 isozymes. However, structural studies so far have had to rely on truncated variants of the enzymes to make conventional X-ray crystallographic and solution-state NMR techniques applicable. In spite of significant efforts it has not yet been possible to crystallize any of these proteins in their full-length membrane bound forms. The main aim of our current research is to demonstrate how advanced modern solid-state NMR spectroscopic techniques will be able to make substantial progress in the structural studies of cyt P450. In fact, I will present results to demonstrate that solid-state NMR is the only technique available today for structural studies on full-length cyt P450 and full-length cyt b5. NMR methods to probe local dynamics, dynamics-based spectral editing techniques on uniformly-labeled cyt b5, and results from b5-P450 complex under physiological conditions will be presented. Suitability of MLVs, bicelles, LUVs, and mechanically-aligned bilayers for solid-state NMR measurements will be discussed. In addition, high-resolution NMR structure, membrane orientation, and functional properties of a variety of amyloid proteins and amyloid peptides will be presented.

For further reading: Cytochrome: J. Am. Chem. Soc., 129, 6670 (2007); BBA Biomembranes 1768, 3235(2007); Angew. Chem., 47, 7864(2008). Amyloid proteins: J.Am. Chem. Soc., 131, 4470(2009); Biochemistry 47, 12689(2008); Biochemistry 47, 12680(2008); J. Am. Chem. Soc., 130, 6424(2008); BBA Biomembranes 1768, 2026(2007). Antimicrobial peptides: Chem. Eur. J., 15 2036(2009); Biochemistry 47, 5565(2008); Biochemistry 47, 9243(2008); ChemBioChem 9, 370(2008); BBA Biomembranes 1778, 357(2008).

\section{SP6-03 分子シミュレーションと中性子散乱で探る蛋白質の立体構造ダイ ナミクス \\ Molecular simulation and neutron scattering studies of protein dynamics}

Yasumasa Joti (1) ((1) Institute of Molecular and Cellular Biosciences, University of Tokyo)

Conformational motions of protein play important roles in its function. Neutron scattering is suitable for studying protein dynamics which occurs on a timescale ranging from femto-seconds to nano-seconds. The "glass-like" transition in protein is observed in elastic incoherent neutron scattering as an increase in the atomic mean-square fluctuation at temperatures greater than $\sim 200 \mathrm{~K}$. It has been argued that protein dynamics at lower temperatures can be nearly regarded as harmonic motions within one of the conformational substates and makes a transition to anharmonic motions that involve jumping among different substates at higher temperatures. Such anharmonic motions have been shown to be crucial to protein function. The glass-like transition is strongly suppressed in dry protein, indicating that solvent molecules are involved with the activation of anharmonic motions. Anharmonic motions of protein have been also detected by quasielastic neutron scattering.

Molecular dynamics simulation is also a powerful technique for studying the protein dynamics. It simulates the detailed motion of all atoms on a timescale ranging from femto-seconds to micro-seconds, which cannot be observed directly by experiments. The combination of simulations with neutron scattering experiments allows us to characterize a wide range of dynamical phenomena in biomolecular systems. 